\title{
Generation of Locomotion Trajectories for Series Elastic and Viscoelastic Bipedal Robots
}

\author{
Alexander Werner, Wojciech Turlej and Christian Ott
}

\begin{abstract}
Series-elastic and viscoelastic robots can provide performance gains in applications with high dynamics. Harnessing these, requires an understanding of the dynamics of the system, which can be gained using optimization-based methods. The result are motions which make optimal use of the intrinsic behavior, possibly exceeding the performance of an equivalent rigid-body robot. We present a collocation framework which enables both automatic computation of contactswitching patterns and allows the full utilization of the dynamics of the compliant system. The formulation also addresses the problem of redundant torque generation in viscoelastic actuators. The effectiveness of this method was demonstrated in simulations as well as experiments with a compliant bipedal robot. The approach is capable of providing gait primitives, longer gait sequences containing multiple steps as well as generating extremely dynamic motions, e.g. somersaults.
\end{abstract}

\section{INTRODUCTION}

The direction of humanoid robotics is towards in combining performance and robustness. Both are clearly linked to hardware design aspects, especially the actuation principle. One direction research has successfully progressed in is the application of series-elastic actuators to robotic legs. The first use in [1] was largely motivated by achieving mechanical robustness in combination with torque sensing. Torque sensing alone can also be achieved without series-elastic actuator (SEA) like concepts; however, it is usually less robust against high peak torques.

To improve performance periodic locomotion can be generated through the intrinsic behavior of a passive mechanism was first explored in [2]. This class of systems shows very low cost of transport, a goal shared by their actuated counterparts. As these robots show, one system Eigenmode can be well matched to a mode of locomotion. But whenever the mode of the system does not exactly match the desired locomotion style, actuation is required. In contrast to rigid-body robots, feasibility of these trajectories is complicated by the second order dynamics of the SEAs.

For robots with multiple degrees of freedom, currently the only way to provide the optimal trajectories is using large scale optimization problems computed offline. Problem formulations differ in the way a solution is parameterized.

All authors are with the Institute for Robotics and Mechatronics, German Aerospace Center (DLR). E-mail address < firstname $>.<$ lastname $>@$ dlr.de. This work was partially supported by the Helmholtz Association (VH-NG-808) and by the European Commission (H2020-ICT-645097 COMANOID).

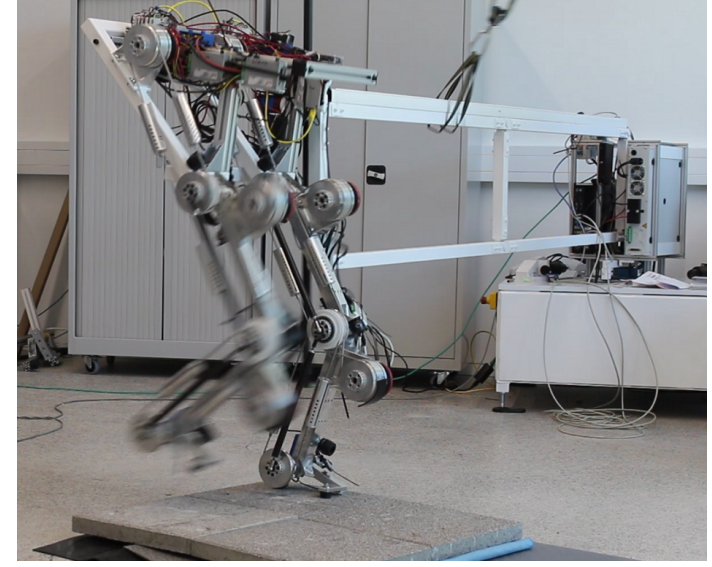

Fig. 1. Series Elastic Biped C-Runner dynamically stepping onto an obstacle $80 \mathrm{~mm}$ high.

Shooting-based methods parameterize the system input, in the case of series-elastic robots the motor torques. This is the most general parameterization and the optimization problem has to implement the least complex constraints. Here, the non-linearity resulting from the changing contacts is hidden in the dynamics. The ODE for the dynamics however is stiff, especially when high contact stiffnesses are implemented. An advantage can be seen when applying this method to rigid-body robots as it allows manipulation of the contact forces rather directly. However achieving a position level task results in the optimization method linearizing the system dynamics. For rigid-body robots this second degree ODE can still be addressed by this method, for series-elastic joints however linearizing a fourth degree ODE is rather problematic. Multiple-shooting [3], [4] partially addresses this by partitioning the integration interval and parameterizing the system states at the partition bounds.

A consistent parameterization of not only the system states but also accelerations on the other hand allows the optimization method to directly solve position level and force level tasks [5]. Contact forces then can be computed using inverse dynamics and constraints imposed on them to render the motion feasible. However any kind of underactuation, which is common in locomotion tasks, cannot be handled. Similar to multiple-shooting, collocation-based methods [6], [7] increase the optimization problem size and parameterize the system inputs as well as system states, allowing the solver to find a solution for the contact forces and the constraints depending on the 
states separately, enforcing an equality constraint to render the trajectory physically consistent. In conventional methods, the contact sequence is defined a-priori. In this work we leave the contact state free for the optimizer by introducing independent parameterization of the contact forces during the complete trajectory. These physically admissible contact forces can only exist when kinematically in contact, which is ensured by additional equality constraints. In another words this allows the optimizer to chose from the different modes of the hybrid system using continuous variables. A consequence of this is that the method is capable of producing human-like gaits without any predefined concept that an efficient gait contains a specific sequence of heel- and toe-phases. To discretize the problem in the time domain, we parameterize all physical states using spline functions.

As we are applying collocation to series-elastic actuators, the natural system input is the motor torque. Using this input in a collocation scheme, however, requires the solver to again linearize the fourth order system, similar to shooting. Motivated by [8], it is acceptable to ignore inertial coupling of the motor and link sides. With this assumption, a reasonable choice is to parameterize the motor states and use inverse dynamics to compute the required motor torques. This reduces the relevant system order to two, allowing better robustness and speed of the method. Knowing the motor states and accelerations allows us to explore not only series-elastic actuators, but also actuators with a viscous damper in parallel to the spring (viscoelastic actuators, SVEA).

This paper is structured as follows: First, we review related methods in Section II followed by the problem statement summarizing the task at hand in III. Then the optimization problem is stated in Section IV. We adapt the initial formulation of the optimization problem as described in Section V. Section VI shows the numerical results and also discusses capabilities of the problem formulation. Section VIII presents our conclusions for the presented method. The following Section VII summarizes the experiments. Our future research intentions are given in Section IX.

\section{RELATED WORK}

Various approaches for generating locomotion trajectories for legged systems have been published. Some approaches focus on the most important dynamics, covered by models with one concentrated mass. Those include the linear inverted pendulum (LIP) and spring-loaded inverted pendulum (SLIP) models. Motions of such a reduced model can either be generated analytically [9], [10] or by using optimization [11]. These methods have been applied to intrinsically compliant bipedal robots [12]. Often this results in conservative use of the robots capabilities, especially in the case of compliant robots. Hence, we are primarily motivated by implementing the non-linear system constraints which is only possible with a high-dimensional model. This enables the same robot to execute more challenging tasks.

Trajectories for these complex models can only be generated using numerical methods. For rigid-body robots, many approaches have been presented [5], [6], [13]. As shown in [14], the application of optimization-based methods is even more advantageous when applied to SEA based robots, as the properties of these robots are much less intuitive. Recently applications in the context of trajectory generation for hybrid zero-dynamics controllers were shown [7].

The ability to generate motions with arbitrary contact points and sequences has been studied in [15]. Mordatch et al. [16] describes a contact-invariant trajectory generation method that utilizes the elastic structure and an implicit contact model to provide complex dynamic trajectories for animated characters. The authors proposed a formulation which models contacts as a complementarity constraint in a collocation-based optimization problem. The motions of the humanoid models used in the paper were parameterized using Cartesian trajectories. Further work of the same authors shows the application of the same method to bipedal locomotion [17] and hand manipulation tasks [18].

Posa et al. [19] proposed an off-line optimization method, also based on the idea of complementarity constraints and have shown its application for simulated biped robots. The authors focused on the optimization of periodic bipedal locomotions trajectories.

Currently no walking robots using SVEA exist to the authors knowledge. However the performance gains of adding intrinsic damping to series elastic actuators are now studied frequently [20], [21]. We extrapolate that this addition will significantly increase the maximum capabilities in comparison to a robot solely based on SEA.

\section{Problem Statement}

Although the method can be easily adapted to the general 3D case, we illustrate the approach using a $2 \mathrm{D}$ model for simplicity. The configuration of the robot is described by

$$
\boldsymbol{y}=\left[\begin{array}{l}
\boldsymbol{x} \\
\boldsymbol{q}
\end{array}\right] \in \mathbb{R}^{N_{D}}
$$

with $\boldsymbol{x} \in \mathbb{R}^{3}$ representing the non-actuated base coordinates, containing the horizontal translation $x_{1}$, vertical translation $x_{2}$ and base link rotation $\phi$, and $\boldsymbol{q} \in \mathbb{R}^{6}$ the joint angles. Fig. 2 describes the coordinates in detail.

The link side dynamics of the robot, which has $N_{D}$ DOF in total of which $N_{J}$ are actuated by elastic actuators, are described by

$$
\boldsymbol{M}(\boldsymbol{y}) \ddot{\boldsymbol{y}}+\boldsymbol{C}(\boldsymbol{y}, \dot{\boldsymbol{y}}) \dot{\boldsymbol{y}}+\boldsymbol{g}(\boldsymbol{y})=\boldsymbol{S}^{T} \boldsymbol{\tau}+\sum_{i=0}^{N_{C}} \boldsymbol{J}_{\mathrm{C}, \mathrm{i}}^{T} \boldsymbol{F}_{\mathrm{C}, \mathrm{i}}
$$

with the inertia matrix $M \in \mathbb{R}^{N_{D} \times N_{D}}$, Coriolis matrix $\boldsymbol{C} \in \mathbb{R}^{N_{D} \times N_{D}}$, gravity terms $\boldsymbol{g} \in \mathbb{R}^{N_{D}}$, joint torques $\boldsymbol{\tau} \in$ 


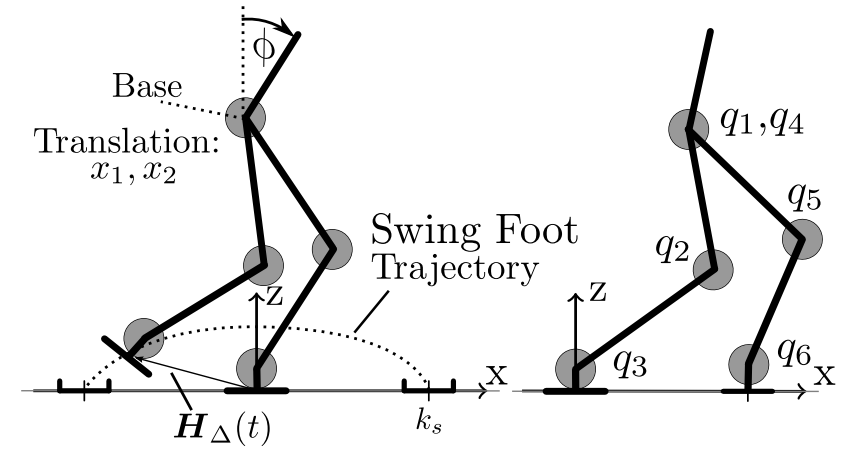

Fig. 2. Kinematic of the planar robot. Left: Schematic motion of the swing foot during the single-support phase with start and goal foot step locations. Right: Closed kinematic Loop during the double-support phase.

$\mathbb{R}^{N_{J}}$, contact point Jacobians $\boldsymbol{J}_{\mathrm{C}, \mathrm{i}} \in \mathbb{R}^{2 \times N_{D}}$, and contact forces $\boldsymbol{F}_{\mathrm{C}, \mathrm{i}} \in \mathbb{R}^{2}$. For the sake of clarity, the dependencies will be omitted for the remainder of the paper. The motor dynamics are coupled to the link side dynamics through linear springs forming an SEA or optionally with nonzero $\boldsymbol{D}$ a SVEA:

$$
\begin{aligned}
\boldsymbol{\tau} & =\boldsymbol{K}(\boldsymbol{\theta}-\boldsymbol{q})+\boldsymbol{D}(\dot{\boldsymbol{\theta}}-\dot{\boldsymbol{q}}) \\
\boldsymbol{B} \ddot{\boldsymbol{\theta}} & =\boldsymbol{\tau}_{m}-\boldsymbol{\tau}
\end{aligned}
$$

where $\boldsymbol{K} \in \mathbb{R}^{N_{J} \times N_{J}}$ is the stiffness matrix, $\boldsymbol{D} \in \mathbb{R}^{N_{J} \times N_{J}}$ is the damping matrix, $\boldsymbol{\theta} \in \mathbb{R}^{N_{J}}$ the motor positions, $\boldsymbol{B} \in \mathbb{R}^{N_{J} \times N_{J}}$ the diagonal motor inertia matrix, and $\boldsymbol{\tau}_{m} \in \mathbb{R}^{N_{J}}$ the motor torques. This formulation neglects inertial coupling between the motor and the link side [8].

Combining (2), (3) and (4) in state space form yields:

$$
\begin{array}{r}
{\left[\begin{array}{c}
\dot{y} \\
\dot{\boldsymbol{y}} \\
\boldsymbol{\theta} \\
\dot{\boldsymbol{\theta}}
\end{array}\right]=\boldsymbol{A}\left[\begin{array}{c}
\boldsymbol{y} \\
\dot{\boldsymbol{y}} \\
\boldsymbol{\theta} \\
\dot{\boldsymbol{\theta}}
\end{array}\right]+\left[\begin{array}{c}
\mathbf{0} \\
\mathbf{0} \\
\mathbf{0} \\
\mathbf{1} / \boldsymbol{B}
\end{array}\right]\left(\boldsymbol{\tau}_{m}-\boldsymbol{\tau}_{f}\right)+\ldots} \\
\ldots+\left[\begin{array}{ccc}
\mathbf{0} & \mathbf{0} & \ldots \\
\boldsymbol{J}_{\mathrm{C}, \mathrm{i}}^{T} & \boldsymbol{J}_{\mathrm{C},(\mathrm{i}+1)}^{T} & \ldots \\
\mathbf{0} & \mathbf{0} & \ldots \\
\mathbf{0} & \mathbf{0} & \ldots
\end{array}\right]\left[\begin{array}{c}
\boldsymbol{F}_{\mathrm{C}, \mathrm{i}} \\
\boldsymbol{F}_{\mathrm{C}, \mathrm{i}+1)} \\
\vdots
\end{array}\right]
\end{array}
$$

$\boldsymbol{A}=\left[\begin{array}{cc|cc}\mathbf{0} & \mathbf{0} & \mathbf{0} & \boldsymbol{I} \\ -\boldsymbol{M}^{-1} \boldsymbol{S}^{T} \boldsymbol{K} & -\boldsymbol{M}^{-1} \boldsymbol{S}^{T} \boldsymbol{D} & \boldsymbol{M}^{-1} \boldsymbol{S}^{T} \boldsymbol{K} & \boldsymbol{M}^{-1} \boldsymbol{S}^{T} \boldsymbol{D} \\ \hline \mathbf{0} & \boldsymbol{I} & \mathbf{0} & \mathbf{0} \\ -\boldsymbol{B}^{-1} \boldsymbol{S}^{T} \boldsymbol{K} & -\boldsymbol{B}^{-1} \boldsymbol{S}^{T} \boldsymbol{D} & \boldsymbol{B}^{-1} \boldsymbol{S}^{T} \boldsymbol{K} & \boldsymbol{B}^{-1} \boldsymbol{S}^{T} \boldsymbol{D}\end{array}\right]$

This formulation can also describe biarticular couplings which do not connect motor and link side. A motivation for these can be found in [22]. It also illustrates the fourth order system dynamics which dominate the problem, especially in systems where $\boldsymbol{D}$ is zero. The eigenvalues of $\boldsymbol{A}$ can give information which frequencies make up the system behavior. However, note that these equations do not contain any contact dynamics which are present in reality. It can be safely assumed that the eigenvalues of the contact dynamics are much faster than all eigenvalues of $\boldsymbol{A}$.

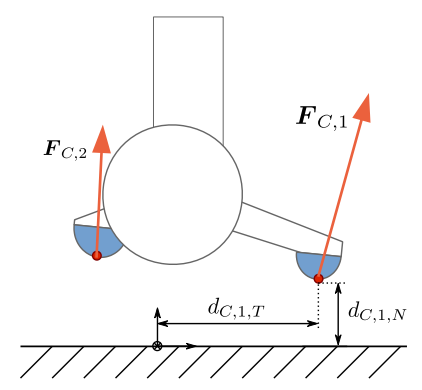

Fig. 3. Contact Forces $\boldsymbol{F}_{1}$ and $\boldsymbol{F}_{2}$ specified for the corresponding contact points on the robot. Normal distance $d_{\mathrm{C}, \mathrm{i}, \mathrm{N}}$ between the contact surface of the contact point 1 . Tangential distance $d_{\mathrm{C}, \mathrm{i}, \mathrm{T}}$ between an arbitrary fixed point on the contact surface and the contact points 1 .

The following inequalities describe contact forces which do not generate slipping and only create unilateral forces

$$
\begin{aligned}
\left|F_{\mathrm{C}, \mathrm{i}, \mathrm{T}}\right| & \leq \mu F_{\mathrm{C}, \mathrm{i}, \mathrm{N}} \\
0 & \leq F_{\mathrm{C}, \mathrm{i}, \mathrm{N}}
\end{aligned}
$$

with the friction coefficient $\mu$, the contact forces component $F_{\mathrm{C}, \mathrm{i}, \mathrm{T}}$ tangential to the contact plane and $F_{\mathrm{C}, \mathrm{i}, \mathrm{N}}$ as the normal component, as illustrated in Fig. 3. As can be seen in the figure, two contact points are defined for each foot, each of which has separate contact forces.

On the kinematic level, link position limits and environment collision constraints have to be ensured:

$$
\begin{aligned}
\boldsymbol{q}_{\min } & \leq \boldsymbol{q} \leq \boldsymbol{q}_{\max } \\
f_{\text {coll }}(\boldsymbol{y}) & \leq \mathbf{0}
\end{aligned}
$$

The physical limits of the actuator can be approximated by the following motor speed and torque constraints

$$
\begin{gathered}
|\dot{\boldsymbol{\theta}}| \leq \dot{\boldsymbol{\theta}}_{\max }, \\
\left|\boldsymbol{\tau}_{m}\right| \leq \boldsymbol{\tau}_{\text {m,max }} .
\end{gathered}
$$

Given the initial system state and the system dynamics, the goal is to find a trajectory of given time $t_{f}$ which achieves a task. Arbitrary tasks can be defined, as an example a center of mass (CoM) position task is used:

$$
\begin{aligned}
& \boldsymbol{x}_{c}(0) \stackrel{!}{=} \boldsymbol{x}_{\mathrm{c}, \mathrm{d}, 0} \\
& \boldsymbol{x}_{c}\left(t_{f}\right) \stackrel{!}{=} \boldsymbol{x}_{\mathrm{c}, \mathrm{d}, \mathrm{f}}
\end{aligned}
$$

where $\boldsymbol{x}_{c}(t)$ is the CoM position, $t_{f}$ the duration of the trajectory and $\boldsymbol{x}_{\mathrm{c}, \mathrm{d}, 0}$ and $\boldsymbol{x}_{\mathrm{c}, \mathrm{d}, \mathrm{f}}$ are the desired CoM position at begining and end of the trajectory. Additionally all velocity and acceleration states should be zero at the final time $t_{f}$ :

$$
\begin{array}{ll}
\dot{\boldsymbol{y}}\left(t_{f}\right)=\mathbf{0} & \ddot{\boldsymbol{y}}\left(t_{f}\right)=\mathbf{0} \\
\dot{\boldsymbol{\theta}}\left(t_{f}\right)=\mathbf{0} & \ddot{\boldsymbol{\theta}}\left(t_{f}\right)=\mathbf{0} .
\end{array}
$$

This trajectory is subject to the cost function

$$
\Gamma=\int_{0}^{t_{f}}\left(\boldsymbol{\tau}_{m}^{T} \dot{\boldsymbol{\theta}}\right)^{2} \mathrm{~d} t
$$


to generate energy efficient trajectories. For locomotion tasks gaits this expresses the cost of transport when the overcome distance is part of the task.

\section{Optimization Problem}

We pose the optimization problem

$$
\begin{array}{r}
\min _{\boldsymbol{p}} \Gamma(\boldsymbol{p}) \\
\boldsymbol{e}(p)=0 \\
\boldsymbol{f}(p)<0
\end{array}
$$

with the cost function $\Gamma(\boldsymbol{p})$, the optimization parameters $\boldsymbol{p}$, the equality constraints $\boldsymbol{e}(\boldsymbol{p})$ and the inequality constraints $\boldsymbol{f}(\boldsymbol{p})$.

The system state is parameterized at the configuration level $\boldsymbol{y}(t)$ using an at least twice-differentiable function. Likewise the contact forces $\boldsymbol{F}_{\mathrm{C}, \mathrm{i}}(t)$ and motor positions $\boldsymbol{\theta}(t)$ are parameterized.

To ensure physical correctness on the link side, (2) must hold. We formulate the vector valued equality constraint

$$
\boldsymbol{e}_{\mathrm{eom}}=\boldsymbol{O}(\boldsymbol{y}, \dot{\boldsymbol{y}}, \ddot{\boldsymbol{y}})-\sum_{i=0}^{N_{C}} \boldsymbol{J}_{\mathrm{C}, \mathrm{i}} \boldsymbol{F}_{\mathrm{C}, \mathrm{i}}-\boldsymbol{S}^{T} \boldsymbol{\tau}
$$

where $\boldsymbol{O}(\boldsymbol{y}, \dot{\boldsymbol{y}}, \ddot{\boldsymbol{y}})$ represents the left hand side of (2). This requires $\boldsymbol{\tau}$ which can be computed using (3) as all dependencies $(\boldsymbol{q}, \dot{\boldsymbol{q}}, \boldsymbol{\theta}, \dot{\boldsymbol{\theta}})$ are known. Additionally, the contact forces must be admissible at this configuration, this is ensured through the complementarity constraints

$$
F_{\mathrm{C}, \mathrm{i}, \mathrm{N}} \cdot d_{\mathrm{C}, \mathrm{i}, \mathrm{N}}+F_{\mathrm{C}, \mathrm{i}, \mathrm{N}} \cdot\left|\dot{d}_{\mathrm{C}, \mathrm{i}, \mathrm{T}}\right|=0
$$

which is a central aspect of the optimization problem. Similar to (15), it removes redundancy in the parameterization of the trajectory. The redundancy here enables the optimization problem to describe arbitrary contact sequences. The tangential forces are automatically constrained by the combination of (8) and (16).

To ensure feasibility of the motion on the robot, the system constraints (8), (9) and (10) are added to the inequalities of the optimization problem. Note that this implies that the trajectories do not contain slipping of contacts. (9) also constrains the contact points not to penetrate the contact surface:

$$
d_{\mathrm{C}, \mathrm{i}, \mathrm{N}} \geq 0 \text {. }
$$

To impose the locomotion task on the optimization problem, (11) and (12) are added to the equality constraints of the optimization problem.

As parameterization function

$$
\boldsymbol{y}=\boldsymbol{f}_{\mathrm{y}}\left(\boldsymbol{p}_{y}, t\right) \quad t \in\left[0 ; t_{f}\right]
$$

a uniform B-Spline with the parameters $\boldsymbol{p}_{y} \in \mathrm{R}^{N_{P y}}$ is used. Equivalently the function $\boldsymbol{F}_{\mathrm{C}, \mathrm{i}}(t)$ with the parameters $\boldsymbol{p}_{C} \in \mathrm{R}^{N_{\mathrm{PC}}}$ and $\boldsymbol{\theta}(t)$ with the parameters $\boldsymbol{p}_{\theta} \in \mathrm{R}^{N_{\mathrm{P} \theta}}$ are discretized. These quantities define the parameter vector of the optimization problem

$$
\boldsymbol{p}=\left[\begin{array}{lll}
\boldsymbol{p}_{y} & \boldsymbol{p}_{C} & \boldsymbol{p}_{\theta}
\end{array}\right] .
$$

To discretize the constraints and cost functions in time, the functions are sampled at a number of $N_{V}$ equidistant checkpoints.

\section{OPTIMIZATION STRATEGY AND IMPROVEMENTS}

The presented optimization problem contains the complementarity constraints (16) which are in general difficult to solve. Multiple improvements and methods for solving such problems were proposed in recent years. Posa et al. described in [19] a similar optimization problem and use SNOPT alongside a few substitutions to improving speed and convergence of the optimization. Mordatch et al. [18] used the LBFGS algorithm, including the constraints as soft constraints in the cost function and run a few phases of differently scaled optimization problems. We decided to use IPOPT [23] as the solver, partially using the improvements suggested by Posa et al. in [19].

Collocation is set up in a way in which the optimization can easily resolve (15) locally at one time in the trajectory, as both sides of the equation are parameterized. The complementarity constraint (16) on the other hand shapes the space of feasible solutions in a complex manner. Even though the non-linearity of this constraint in the parameter space is limited, the decision of attaching contacts is complex, especially as there are multiple contact points per foot. From intuition this would create local minima, however we did not experience this to a significant extent.

Taking the ideas of collocation one step further, it is also possible to temporarily relax the decision on the contact sequence. By adapting the complementarity constraint (16) to

$$
F_{\mathrm{C}, \mathrm{i}, \mathrm{N}} \cdot \hat{d}_{\mathrm{C}, \mathrm{i}, \mathrm{N}}+F_{\mathrm{C}, \mathrm{i}, \mathrm{N}} \cdot\left|\dot{d}_{\mathrm{C}, \mathrm{i}, \mathrm{T}}\right|=0
$$

with the new variable $\hat{d}_{\mathrm{C}, \mathrm{i}, \mathrm{N}}$ introduced for the normal contact distance. This variable is added as an additional parameter to the optimization problem and the additional equality constraint is added

$$
\hat{d}_{\mathrm{C}, \mathrm{i}, \mathrm{N}}-d_{\mathrm{C}, \mathrm{i}, \mathrm{N}}=0
$$

to remove the redundancy again. This allows the solver to converge to a solution quicker than without the additional variable. This can also be understood in such a way that this adaptation basically decouples the kinematic non-linearities from the contact decision problem. The idea can also be repeated for $d_{\mathrm{C}, \mathrm{i}, \mathrm{T}}$ with the same motivation.

\section{Numerical Results}

The robot used for the results in this section is described further in Section VII. The initial guess trajectory puts the robot in a time-constant collision-free configuration in contact with the ground, unless otherwise stated. The motor positions $\boldsymbol{\theta}(t)$ are set to $\boldsymbol{q}(0)$, generating no torque, and contact forces $\boldsymbol{F}_{\mathrm{C}, \mathrm{i}}$ are set 


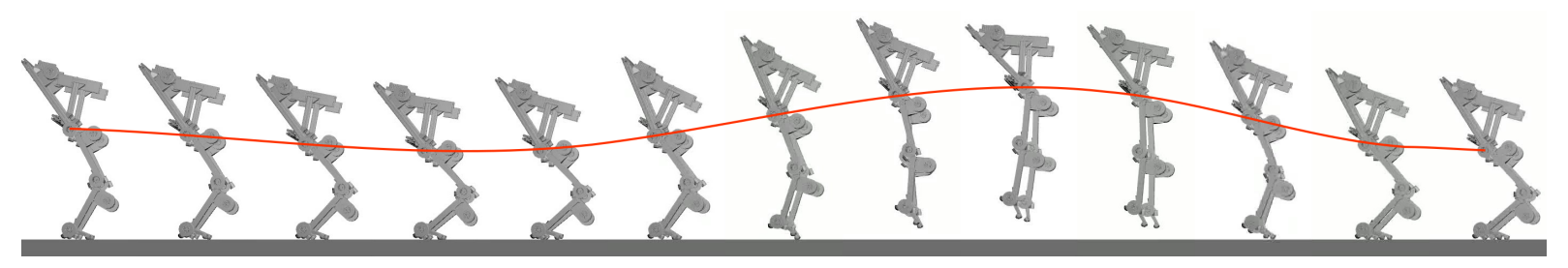

Fig. 4. Snapshots of a trajectory which achieves maximum jump height. The red line denotes the CoM trajectory. During the initial phase it is clearly visible how the link side dynamics are used. At take-off the toe contact is used in this optimal solution as expected.

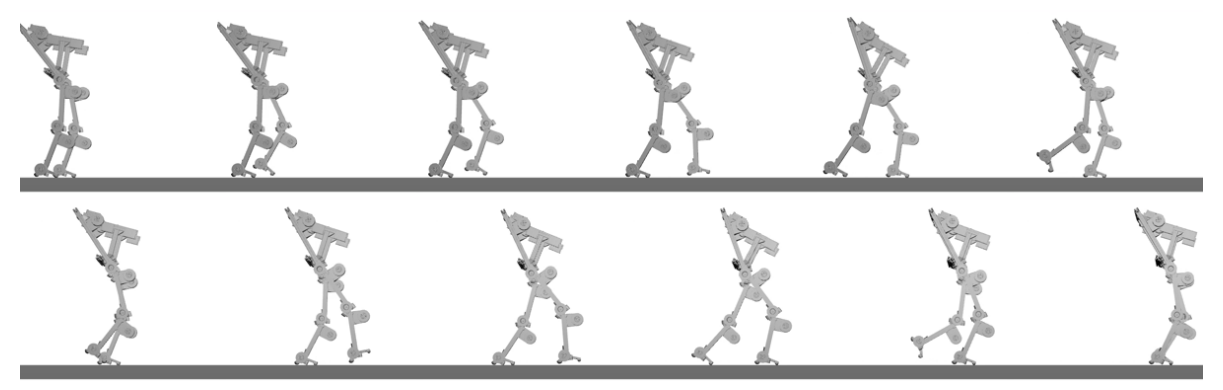

Fig. 5. Snapshots of a multi-step walking trajectory which display the capability of generating human like walking. This trajectory start and end with a static configuration. The execution of this trajectory on the robot is documented in the video attached to the paper.

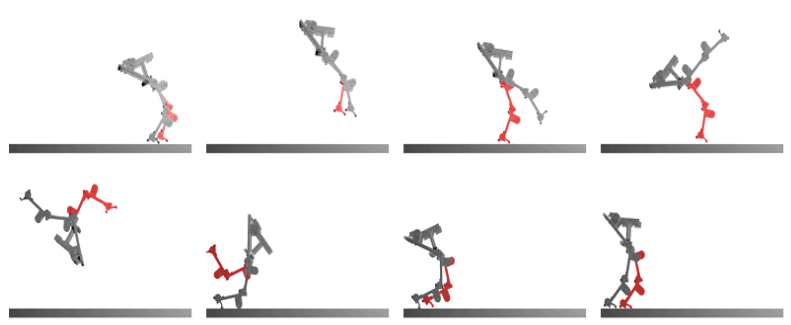

Fig. 6. Somersault trajectory created with the presented method. This highly dynamics result can be achieved with this robot, only with twice the currently available motor torque.
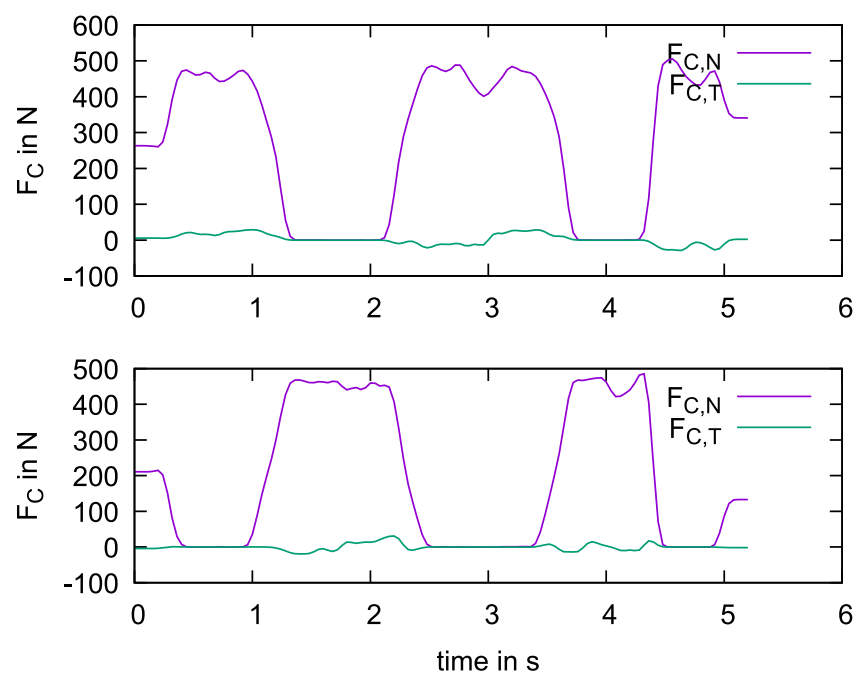

Fig. 7. Contact Forces for a multi-step trajectory. to zero. For the different tasks, the duration $t_{f}$ of the trajectory was chosen heuristically.

In order to achieve static initial and final pose, we enforced the constraints (12) implicitly on the spline [5]. Additionally, the cost function was augmented with a small regularization term on the contact forces to remove internal forces.

The contact sequence is automatically chosen by the proposed scheme. To verify that the sequence of contacts, contact timing and point of contact both on the robot and in the environment are chosen in an appropriate manner, we set up the task of walking a fixed distance, within a fixed time, on flat ground. Initially a trajectory time of $t_{\mathrm{f}}=5.2 \mathrm{~s}$ was used and then motion was parameterized with 66 free parameters for fourth order B-Splines per degree of freedom $N_{\mathrm{D}}+N_{\mathrm{J}}+3 \cdot N_{\mathrm{C}}$. Three splines were used to parameterize the normal, the tangential forces and the normal distance (see (20)) per contact point. The resulting problem dimension was 1782 .

To ensure feasibility of the motion, constraints which are functions of time were evaluated at discretization intervals of $\Delta t=0.04 \mathrm{~s}$. For defining the locomotion task in the optimization problem, the positions of the feet in sagital direction were constrained to be between $-0.1 \mathrm{~m}$ and $0 \mathrm{~m}$ at $t=0 \mathrm{~s}$ (allowing for split stance), and between $1.6 \mathrm{~m}$ and $1.8 \mathrm{~m}$ at $t_{\mathrm{f}}$. Additionally, the sagital position of the base was constrained between the feet at the end of the task. We have chosen to constrain these quantities rather than just enforcing a stable COM position to yield more robust initial and final poses, thereby increasing the error margin when conducting experiments on the robot.

The optimisation resulted in smooth, natural-looking motion with heel- and toe phases. Using 5 steps the 

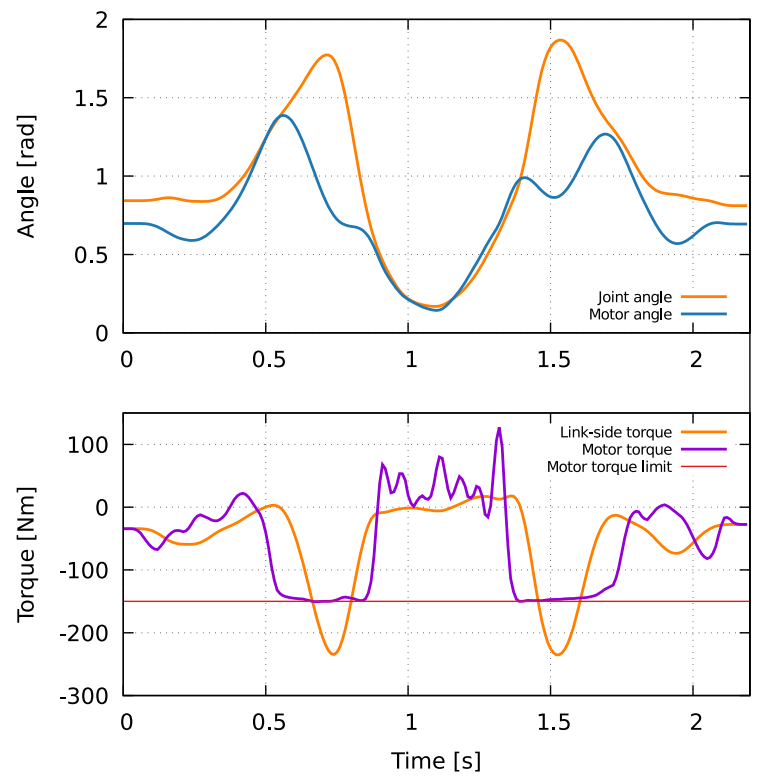

Fig. 8. Quantities for a high jump trajectory. Top: Motor and joint angle for the knee. Bottom: Motor and link torque for the knee. The red line denotes the maximum motor torque $\boldsymbol{\tau}_{\mathrm{m}, \max }$.

solution displayed a gait initiation phase and a stopping motion at the end. Note that whole motion was produced in one optimization run, and the number of steps, as well as contact timing and positions are all parameterized in this optimization problem. There were no constraints or heuristics provided for ensuring the periodic nature of the walking motion, which emerged automatically during the optimization. Fig. 5 illustrates the trajectory.

In order to investigate capabilities of described method, we ran further set of experiments, where, while all other parameters were kept, we varied the distance and time in equal measure. The method then produced trajectories containing the appropriate number of steps, adding more steps as necessary. The video attached to the paper illustrates the solutions found for the different tasks. This leads to natural ground reaction force illustrated in Fig. 7.

The chosen contact sequence is established early on in the optimization process. This leads to local minima in trajectories which contain multiple steps. Especially present is this behavior in open problems where the start and final system state is not fully specified. On the other hand adaptation of the contact timing does not seem to be any problem.

Intrinsic dynamics are used by the method whenever possible. To illustrate this, we selected the task of highjumping. Jumping motions was defined as a task constraint that the base has to reach at least $1.5 \mathrm{~m}$ over the ground in the middle of the motion. The resulting trajectory is shown in Fig. 4. Fig. 8 highlights how the intrinsic dynamics are used to generate a jump. In this particular case the motor inertia is accelerated before the jump to provide a torque in excess of what can be generated by the motor.

Acrobatic trajectories can be produced, e.g. somersaults. The task was specified as abstract as possible, in form of the requirement to jump (lift the base) and end up in a stable configuration. The flip motion was induced by setting the initial guess to a full rotation of the base. In the trajectory found, the optimizer produced a natural motion of the feet and distributed the forces well. This motion was produced with the system configuration described in Section VII, however $\boldsymbol{\tau}_{\max }$ had to be increased by a factor two, otherwise no solution was found. The trajectories are illustrated in Fig. 6.

\section{Experimental Results}

On the planar SEA robot C-Runner we evaluated a multi-step walking trajectory, stepping up an obstacle and jumping on the spot. Hardware details about the robot are available in [22]. C-Runner is a planar walking robot with modular actuation and a weight of $67 \mathrm{~kg}$. For the experiments in this paper the actuators were SEAs. The base of the robot is free to move in three directions while the other directions are constrained using a rotating boom which guides the robot. The robot has six actuated joints and two feet. This enables balancing on the spot which is a necessity for any real world walking robot.

For comparing this robot to other systems it is most useful to look at the characteristic numbers regarding actuation of C-Runner: the maximum instantaneous motor torque is $400 \mathrm{Nm}$, the maximum motor velocity $5.2 \mathrm{rad} / \mathrm{s}$, projected motor inertia of $1.62 \mathrm{~kg} \cdot \mathrm{m}^{2}$, and the spring stiffnesses of $451 \mathrm{Nm} / \mathrm{rad}, 560 \mathrm{Nm} / \mathrm{rad}$ and $423 \mathrm{Nm} / \mathrm{rad}$ for the hip, knee and ankle joints respectively. The admissible link side torque is limited to $200 \mathrm{Nm}$. The link side inertia in the hip joint for a typical configuration is $2.7 \mathrm{~kg} \cdot \mathrm{m}^{2}$. At the time of experiment, no intrinsic damping (SVEA) was implemented on the system. To increase experiment reliability, conservative values were used to parameterize the contact forces and the motor torque constraints.

We used the following controller to execute the trajectories:

$$
\begin{aligned}
\boldsymbol{\tau}_{m} & =\boldsymbol{K}_{P}\left(\boldsymbol{\theta}_{d}-\boldsymbol{\theta}\right)+\boldsymbol{K}_{D}\left(\dot{\boldsymbol{\theta}}_{d}-\dot{\boldsymbol{\theta}}\right) \\
\boldsymbol{\theta}_{d} & =\boldsymbol{q}+\boldsymbol{K}^{-1} \boldsymbol{\tau}_{d} \\
\boldsymbol{\tau}_{d} & =\boldsymbol{K}\left(\boldsymbol{q}_{T}-\boldsymbol{q}\right)+\boldsymbol{D}\left(\dot{\boldsymbol{q}}_{T}-\dot{\boldsymbol{q}}\right)+\boldsymbol{\tau}_{T}
\end{aligned}
$$

with the diagonal gain matrices $\boldsymbol{K}_{P}, \boldsymbol{K}_{D}$ for the motor position controller. The desired motor position $\boldsymbol{\theta}_{d}$ was computed to realize a compliant behavior on the link side, defined by $\tau_{d}$ which replicates the intrinsic stiffness $\boldsymbol{K}$ of the system in the control law and augments it with the damping matrix $\boldsymbol{D}$. The trajectory defines $\boldsymbol{q}_{T}$, $\dot{\boldsymbol{q}}_{T}$ and $\boldsymbol{\tau}_{T}$. Note that this controller does not explicitly implement any balancing behavior but is used in a feedforward manner.

As an example of the multi-step trajectory generation from the optimizer, we created a walking motion with 4 


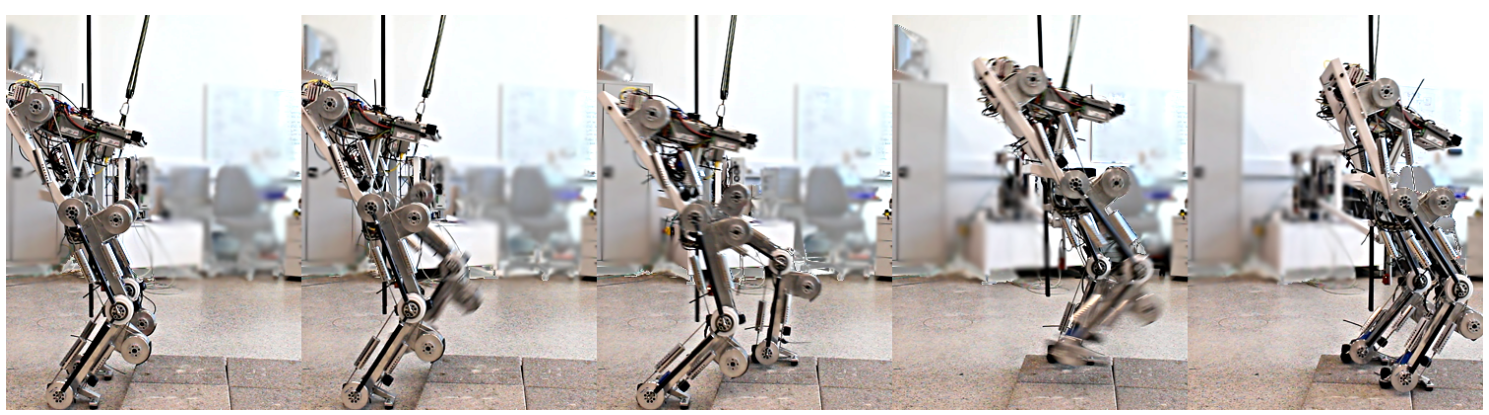

Fig. 9. Snapshots of a trajectory for stepping of a $80 \mathrm{~mm}$ obstacle. The robot climbs the obstacle in a dynamics way. In the final configuration the feet are split to provide a larger support polygon. This is necessary as the used control approach does not stabilize the robot.

steps. This trajectory shows the capability of the solver to produce a continuous gait, including an acceleration phase. As the control approach provided no stabilization of the floating base $\boldsymbol{x}$, a minimal stabilization was generated by a human applying a force of approximately $20 \mathrm{~N}$ to the boom constraining the robot. Fig. 7 illustrates the planned contact forces during the gait. The experiments are also shown in the video attached to the paper. During the walking experiment the robot displays some oscillations in the joint space. This can be attributed to a combination of model error and the low amount of damping that can be generated by the controller used.

As show in Fig. 1 the method can also easily be adapted to any other task. This motion of stepping up one step especially shows the dynamic trajectories of the approach.

\section{Conclusions}

The described method is able to provide locomotion trajectories for the targeted system class of underactuated robots with hybrid dynamics in form of switching contacts. The method addresses the underactuation of the base, as well as the underactuation given by the elastic actuators.

As a collocation method, it splits the forth order system dynamics in the middle on the level of link torque. This presents the problem in a way to the solver where the optimization of the elastic actuator is a small challenge.

The hybrid aspect is also addressed via collocation with the rigid body side and the contact forces both being parameterized. This allows the method to find the contact sequence automatically. However this creates local minima in form of different contact sequences for problems where multiple steps are required.

This can also be seen as a result of the complementarity constraint (16), which deals with the hybrid aspect of the system. We found this formulation of the complementarity constraint especially useful as the gradients always expose the possibility to make contact, even if the foot is in the air.

For the parameterization of the link side motion through $f_{y}$ we used fourth order splines. Two aspects need to be considered for the spline order. First, given by the system dynamics we know that in parts of the trajectory without contact change, the link side motion for this model is a four times differentiable function. However, higher spline order lowers the sparsity of the optimization problem, making it more difficult to solve. Also, during contact change, the spline ideally has to follow trajectories with discontinuous velocity which would require a zero-order spline (continuous only on position level). However, these functions can only model discontinuities at segment boundaries.

As the optimization problem ensures (15) only at discrete points in time, given the limitations of the spline path quickly results in (15) being slightly violated between those points. This results in the optimizer not being able to move this violation across the checkpoints. One solution to help this is to relax the condition (15) to an inequality constraint with reasonable tolerances. Another option is mentioned in Section IX.

In contrast to our constrained inverse dynamics based optimization methods presented earlier [14], this collocation based method parameterized the contact forces directly. This allow to have multiple contact points on the foot which enable the solver to find heel and toe contact phases.

Computation times vary depending on problem size, but the contact decision is resolved quickly. Given a feasible problem, the solution can usually be found under an hour for a multi-step trajectory. Termination problems of the solver, if present, can be attributed to the limited dynamics the spline can represent. This can be resolved by the relaxation described above.

\section{Future Work}

As previously discussed, we want to improve the solution correctness by adapting the parameterization function to allow a more precise solution at points with highly dynamic motions, e.g. impacts. Using non-uniform B-Splines this can be done by locally adding more or even repeating knot points while ensuring position level continuity. This allows the correct parameterization of discontinuous velocities while keeping the problem size small. 
Application-wise, the first use of this method was to establish which dynamic motions are feasible with the current system limits, suggesting improvements for the robots' actuation design. Now the focus will be set on the generation of both walking and running primitives as well as generation of explosive dynamics motions.

\section{REFERENCES}

[1] J. E. Pratt and B. T. Krupp, "Series elastic actuators for legged robots," in Defense and Security. International Society for Optics and Photonics, 2004, pp. 135-144.

[2] T. McGeer et al., "Passive dynamic walking," I. J. Robotic Res., vol. 9, no. 2, pp. 62-82, 1990.

[3] K. D. Mombaur, H. G. Bock, J. Schloder, and R. W. Longman, "Human-like actuated walking that is asymptotically stable without feedback," in Robotics and Automation, 2001. Proceedings 2001 ICRA. IEEE International Conference on, vol. 4. IEEE, 2001, pp. 4128-4133.

[4] A. Hereid, C. M. Hubicki, E. A. Cousineau, J. W. Hurst, and A. D. Ames, "Hybrid zero dynamics based multiple shooting optimization with applications to robotic walking," in Robotics and Automation (ICRA), 2015 IEEE International Conference on. IEEE, 2015, pp. 5734-5740.

[5] A. Werner, R. Lampariello, and C. Ott, "Optimization-based generation and experimental validation of optimal walking trajectories for biped robots," in 2012 IEEE/RSJ International Conference on Intelligent Robots and Systems, IROS 2012, Vilamoura, Algarve, Portugal, October 7-12, 2012, 2012, pp. 4373-4379.

[6] T. Buschmann, S. Lohmeier, M. Bachmayer, H. Ulbrich, and F. Pfeiffer, "A collocation method for real-time walking pattern generation," in Humanoid Robots, 2007 7th IEEE-RAS International Conference on. IEEE, 2007, pp. 1-6.

[7] A. Hereid, E. A. Cousineau, C. M. Hubicki, and A. D. Ames, "3d dynamic walking with underactuated humanoid robots: A direct collocation framework for optimizing hybrid zero dynamics," in Robotics and Automation (ICRA), 2016 IEEE International Conference on. IEEE, 2016, pp. 1447-1454.

[8] M. Spong, "Modelling and control of robots with elastic joints," Journal Dynamic Systems, Measurement and Control, vol. 109, pp. 310-319, 1987.

[9] G. Garofalo, C. Ott, and A. Albu-Schäffer, "Walking control of fully actuated robots based on the bipedal slip model," in Robotics and Automation (ICRA), 2012 IEEE International Conference on. IEEE, 2012, pp. 1456-1463.

[10] P. M. Wensing and D. E. Orin, "3d-slip steering for high-speed humanoid turns," in 2014 IEEE/RSJ International Conference on Intelligent Robots and Systems. IEEE, 2014, pp. 4008-4013.
[11] I. Mordatch, M. de Lasa, and A. Hertzmann, "Robust PhysicsBased Locomotion Using Low-Dimensional Planning," ACM Transactions on Graphics, vol. 29, no. 3, 2010.

[12] I. Poulakakis, "Spring loaded inverted pendulum embedding: Extensions toward the control of compliant running robots," in Robotics and Automation (ICRA), 2010 IEEE International Conference on. IEEE, 2010, pp. 5219-5224.

[13] J. Denk and G. Schmidt, "Synthesis of walking primitive databases for biped robots in 3d-environments," in Robotics and Automation, 2003. Proceedings. ICRA'03. IEEE International Conference on, vol. 1. IEEE, 2003, pp. 1343-1349.

[14] A. Werner, R. Lampariello, and C. Ott, "Trajectory optimization for walking robots with series elastic actuators," in 53rd Conference on Decision and Control $(C D C)$. IEEE, Dec. 2014, pp. 2964-2970.

[15] I. Mordatch, M. De Lasa, and A. Hertzmann, "Robust physicsbased locomotion using low-dimensional planning," in $A C M$ Transactions on Graphics (TOG), vol. 29. ACM, 2010, p. 71.

[16] I. Mordatch, E. Todorov, and Z. Popović, "Discovery of complex behaviors through contact-invariant optimization," $A C M$ Transactions on Graphics (TOG), vol. 31, no. 4, p. 43, 2012.

[17] I. Mordatch, J. M. Wang, E. Todorov, and V. Koltun, "Animating human lower limbs using contact-invariant optimization," ACM Transactions on Graphics (TOG), vol. 32, no. 6, p. 203, 2013.

[18] I. Mordatch, Z. Popović, and E. Todorov, "Contact-invariant optimization for hand manipulation," in Proceedings of the ACM SIGGRAPH/Eurographics symposium on computer animation. Eurographics Association, 2012, pp. 137-144.

[19] M. Posa and R. Tedrake, "Direct trajectory optimization of rigid body dynamical systems through contact," in Algorithmic foundations of robotics $X$. Springer, 2013, pp. 527-542.

[20] M. Laffranchi, N. Tsagarakis, and D. G. Caldwell, "A compact compliant actuator (compact ${ }^{\mathrm{TM}}$ ) with variable physical damping," in Robotics and Automation (ICRA), 2011 IEEE International Conference on. IEEE, 2011, pp. 4644-4650.

[21] M. J. Kim, A. Werner, F. C. Loeffl, and C. Ott, "Joint torque control capability of series elastic actuators with physical damping," in Robotics and Automation (ICRA), 2017 IEEE International Conference on (accepted). IEEE, 2017.

[22] F. Loeff, A. Werner, D. Lakatos, J. Reinecke, S. Wolf, R. Burger, T. Gumpert, F. Schmidt, C. Ott, M. Grebenstein, et al., "The DLR c-runner: Concept, design and experiments," in Humanoid Robots (Humanoids), 2016 IEEE-RAS 16th International Conference on. IEEE, 2016, pp. 758-765.

[23] A. Wächter and L. T. Biegler, "On the implementation of an interior-point filter line-search algorithm for large-scale nonlinear programming," Mathematical Programming, vol. 106, pp. $25-57,2006$. 